\title{
Probability of all eigenvalues real for products of standard Gaussian matrices
}

\author{
Peter J. Forrester \\ Department of Mathematics and Statistics, The University of Melbourne, Victoria 3010, Australia \\ email: p.forrester@ms.unimelb.edu.au
}

\begin{abstract}
With $\left\{X_{i}\right\}$ independent $N \times N$ standard Gaussian random matrices, the probability $p_{N, N}^{P_{m}}$ that all eigenvalues are real for the matrix product $P_{m}=X_{m} X_{m-1} \cdots X_{1}$ is expressed in terms of an $N / 2 \times N / 2(N$ even $)$ and $(N+1) / 2 \times(N+1) / 2(N$ odd) determinant. The entries of the determinant are certain Meijer $G$-functions. In the case $m=2$ high precision computation indicates that the entries are rational multiples of $\pi^{2}$, with the denominator a power of 2 , and that to leading order in $N p_{N, N}^{P_{m}}$ decays as $(\pi / 4)^{N^{2} / 2}$. We are able to show that for general $m$ and large $N$, $p_{N, N}^{P_{m}} \sim b_{m}^{N^{2}}$ with an explicit $b_{m}$. An analytic demonstration that $p_{N}^{P_{m}} \rightarrow 1$ as $m \rightarrow \infty$ is given.
\end{abstract}

\section{Introduction}

The topic of products of random matrices saw much progress in the two decades up to the mid 1980's. The achievements of this era are summarized in the books [13, 20], as well as some articles in the conference proceedings [17]. Interest in the topic seemed to die down somewhat for the subsequent two decades, until in the last few years when a number of researchers, most with backgrounds in integrability properties of the eigenvalue spectrum of large random matrices, have revisited this topic. This has seen the discovery of rich mathematical structures, analogous to those known for certain classes of ensembles of single random matrices, for ensembles of products of random matrices.

The products may be infinite - in which case the quantity of interest is the Lyapunov spectrum [25, 32, — or finite but allowing for an arbitrary number [14, 15, 37, 2, 28, 4, 6. 3. 33, 1, 29]. The random matrices being multiplied typically have Gaussian entries, and an exception being one of the ensembles considered in [1], which involves products of sub-blocks of unitary random matrices. Thus the setting is different to that of random matrix products as they occur in the study of disordered chains [39], or the one-dimensional Anderson model [19, 18] where one typically encounters products of random $2 \times 2$ matrices, some elements of which are fixed. 
The study of integrability properties of the spectrum of a product of two (rectangular) Gaussian matrices was first undertaken by Osborn [35] (see also [31]) in the case of complex entries. This was then generalized by Akemann et al. [5] to the case of real entries. Edelman et al. 23] found a number of exact results associated with the eigenvalues of the product $Y^{-1} X$ for $X, Y N \times N$ real Gaussian matrices. This study was subsequently extended by Forrester and Mays [26].

For $X$ a square real random matrix there is a (typically) non-zero probabiliy $p_{N, k}^{X}$ of their being exactly $k$ real eigenvalues. Since the complex eigenvalues occur in complex conjugate pairs, this requires $k$ to have the same parity as $N$. It was shown in [26] that for the random matrix product $Y^{-1} X$ the probability that all eigenvalues are real is given by

$$
p_{N, N}^{Y-1} X=\frac{\left(\Gamma((N+1) / 2)^{N}\right.}{G(N+1)},
$$

where $G(N+1):=\prod_{l=1}^{N-1} l !,\left(N \in \mathbb{Z}^{+}\right)$is the Barnes- $G$ function. This has the large $N$ form [9]

$$
p_{N, N}^{Y^{-1} X}=N^{1 / 12}\left(\frac{e}{4}\right)^{N^{2} / 4} e^{-\zeta^{\prime}(-1)-1 / 12}\left(1+O\left(N^{-1}\right)\right) .
$$

In the work [9] the probability $p_{N, N}^{Y^{-1} X}$ was shown to have an interpretation relating to the ranks of certain random tensors.

In the case of a single $N \times N$ real Gaussian matrix $X$, a result of Edelman [22] gives that

$$
p_{N, N}^{X}=2^{-N(N-1) / 4}
$$

(see also [30] and [24, \$15.10]). Both (1.2) and (1.3) exhibit a leading order Gaussian decay, but with a slower rate for $p_{N, N}^{Y^{-1} X}$, the corresponding bases being $(e / 4)^{(1 / 4)}$ and $(1 / 2)^{(1 / 4)}$ for $Y^{-1} X$ and $X$ respectively. A recent numerical study of Lakshminarayan [34, motivated by a problem in quantum entanglement, has considered the real eigenvalues for the matrix product

$$
P_{m}=X_{m} X_{m-1} \cdots X_{1},
$$

where each $X_{i}$ is an $N \times N$ is a real standard Gaussian matrix. It was demonstrated that for $N$ fixed the probability of all eigenvalues being real increases as $m$ increases, and approaches 1 . It is the purpose of the present paper to investigate this phenomenon and related questions analytically, using theory developed in the very recent work [1], together with methods familiar from the study of $p_{N, k}^{X}$ in [27]. 


\section{Real eigenvalues of products of real Gaussian ma- trices}

\section{$2.1 \quad$ Determinant formulas}

With each entry of the $N \times N$ matrix $X_{l}$ an independent Gaussian, the probability measure associated with $X_{1}, X_{2}, \ldots, X_{m}$ is equal to

$$
\left(\frac{1}{2 \pi}\right)^{m N^{2} / 2} \prod_{l=1}^{m} e^{-\frac{1}{2} \operatorname{Tr} X_{l} X_{l}^{T}}\left(d X_{l}\right),
$$

where, with $X_{l}:=\left[x_{j, k}^{(l)}\right]_{j, k=1, \ldots, m},\left(d X_{l}\right):=\prod_{j, k=1}^{m} d x_{j, k}^{(l)}$. In the case $m=1$, the key [22] to computing the corresponding eigenvalue distribution is the real Schur decomposition

$$
X=Q R Q^{T}
$$

where $Q$ is an $N \times N$ orthogonal matrix with elements of the first row positive while

$$
R=\left[\begin{array}{cccccc}
\lambda_{1} & \cdots & R_{1, k} & R_{1, k+1} & \cdots & R_{1, m} \\
& \ddots & \vdots & \vdots & \cdots & \vdots \\
& & \lambda_{k} & R_{k, k+1} & \cdots & R_{k, m} \\
& & & Z_{k+1} & \cdots & R_{k+1, m} \\
& & & & \ddots & \vdots \\
& & & & & Z_{m}
\end{array}\right] .
$$

Here all elements not explicitly shown are zero, $m=(N+k) / 2$, and $R_{i j}$ is of size $p \times q$ with

$$
p \times q= \begin{cases}1 \times 1 & \text { if } i \leq k, j \leq k \\ 1 \times 2 & \text { if } i \leq k, j>k \\ 2 \times 1 & \text { if } i>k, j \leq k \\ 2 \times 2 & \text { if } i>k, j>k\end{cases}
$$

The variables $\left\{\lambda_{j}\right\}_{j=1, \ldots, k}$ are the real eigenvalues of $X$, while each $Z_{j}$ is the $2 \times 2$ matrix

$$
Z_{j}=\left[\begin{array}{cc}
x_{j} & b_{j} \\
-c_{j} & x_{j}
\end{array}\right], \quad b_{j}, c_{j}>0
$$

where $x_{j}$ is the real part of the $j$-th complex eigenvalue of $X$ and $y_{j}=\sqrt{b_{j} c_{j}}$ with $y_{j}$ the imaginary part of the $j$-th complex eigenvalue. In the special case $k=N$ the structure of (2.3) thus simplifies and we have

$$
R=\operatorname{diag}\left(\lambda_{1}, \ldots, \lambda_{N}\right)+T
$$

where $T$ is the strictly upper triangular $N \times N$ matrix with non-zero entries $t_{j k}, k>j$. 
Following an idea of Osborn 35] in the complex case with $m=2$, and extended in [2, 1] in the real case for general $m$, for real square matrices $\left\{X_{i}\right\}_{i=1, \ldots, m}$ the real Schur decomposition (2.2) admits the generalization

$$
X_{i}=Q_{i} R_{i} Q_{i+1}^{T} \quad(i=1, \ldots, m)
$$

with $Q_{m+1}:=Q_{1}$. Each $Q_{i}$ is an $N \times N$ orthogonal matrix with elements of the first row positive, and each $R_{i}$ has the structure (2.5). Our task is to use (2.6) to change variables in (2.1) for the sector $k=N$ (all eigenvalues real) then to integrate over all variables except the eigenvalues of $P_{m}$. This will give us $p_{N, N}^{P_{m}}$.

Proposition 1. Let

$$
w_{m}(\lambda)=\left(\frac{1}{\sqrt{2 \pi}}\right)^{m} \int e^{-\sum_{l=1}^{m} x_{l}^{2} / 2} \delta\left(\lambda-\prod_{l=1}^{m} x_{l}\right) d x_{1} \cdots d x_{m}
$$

and let $L$ denote the region

$$
\lambda_{1}>\lambda_{2}>\cdots>\lambda_{N}
$$

We have

$$
p_{N, N}^{P_{m}}=2^{-m N(N+1) / 4}\left(\prod_{j=1}^{N} \frac{1}{\Gamma(j / 2)}\right)^{m} \int_{L} \prod_{j=1}^{N} w_{m}\left(\lambda_{l}\right) \prod_{1 \leq j<k \leq N}\left(\lambda_{j}-\lambda_{k}\right) d \lambda_{1} \cdots d \lambda_{N} .
$$

Proof. We know from the working of [1, Appendix A] that in the case each $R_{i}$ in (2.2) with appropriate subscripts is given by (2.5)

$$
\prod_{l=1}^{m}\left(d X_{l}\right)=\prod_{1 \leq j<k \leq N}\left(\lambda_{j}-\lambda_{k}\right) \prod_{l=1}^{m}\left(d T_{l}\right)\left(Q_{l}^{T} D Q_{l}\right) d \lambda_{l},
$$

where $\left(Q_{l}^{T} D Q_{l}\right)$ denotes the Haar measure on the space of orthogonal matrices with all entries in the first row positive, and $\left\{\lambda_{j}\right\}$ the eigenvalues of $P_{m}$. Furthermore substituting (2.5) for each $R_{i}$ in (2.2) shows

$$
\prod_{l=1}^{m} e^{-\frac{1}{2} \operatorname{Tr} X_{l} X_{l}^{T}}=\prod_{l=1}^{m} e^{-\frac{1}{2} \sum_{k=1}^{N}\left(\lambda_{k}^{(l)}\right)^{2}} e^{-\sum_{j<k}\left(r_{j k}^{(l)}\right)^{2}} .
$$

Substituting (2.9) and (2.10) in (2.1) we see that the dependence on the eigenvalues and the auxiliary variables factorizes. The integrations over the auxiliary variables can be carried out according to [24, second displayed equation below (15.211)]

$$
\int\left(Q^{T} d Q\right)=\pi^{N(N+1) / 4} \prod_{j=1}^{N} \frac{1}{\Gamma(j / 2)}
$$


and

$$
\int e^{-\sum_{j<k}\left(r_{j k}^{(l)}\right)^{2}}\left(d T_{l}\right)=(2 \pi)^{N(N-1) / 4}
$$

The result (2.8) now follows by noting that $\lambda_{k}=\prod_{p=1}^{m} \lambda_{k}^{(p)}$.

The weight function (2.7) is precisely the distribution of the product of $m$ standard Gaussian random variables, to be denoted $\mathrm{N}^{(m)}[0,1]$. It is well known (see e.g. [2] and references therein) that this can be written as an inverse Mellin transform

$$
w_{m}(\lambda)=\frac{1}{(2 \pi)^{m / 2}} \frac{1}{2 \pi i} \int_{c-i \infty}^{c+i \infty}\left(\frac{\lambda^{2}}{2^{m}}\right)^{-s} \Gamma^{m}(s) d s, \quad c>0 .
$$

Introducing the Meijer $G$-function

$$
G_{p, q}^{m, n}\left(z \mid \begin{array}{c}
a_{1}, \ldots, a_{p} \\
b_{1}, \ldots, b_{q}
\end{array}\right)=\frac{1}{2 \pi i} \int_{C} \frac{\prod_{j=1}^{m} \Gamma\left(b_{j}-s\right) \prod_{j=1}^{n} \Gamma\left(1-a_{j}+s\right)}{\prod_{j=m+1}^{q} \Gamma\left(1-b_{j}+s\right) \prod_{j=n+1}^{p} \Gamma\left(a_{j}-s\right)} z^{s} d s
$$

for an appropriate contour $C$, this can be written

$$
w_{m}(\lambda)=\frac{1}{(2 \pi)^{m / 2}} G_{0, m}^{m, 0}\left(\left.\left(\frac{\lambda^{2}}{2^{m}}\right)\right|_{0, \ldots, 0}\right) .
$$

For $m=2$ we have the alternative expression in terms of the $K_{0}$ Bessel function

$$
w_{2}(\lambda)=\frac{1}{\pi} K_{0}(|\lambda|)
$$

It is furthermore the case that $p_{N, N}^{P_{m}}$ can be written as a determinant.

Proposition 2. We have

$$
p_{N, N}^{P_{m}}=2^{-m N(N+1) / 4}\left(\prod_{j=1}^{N} \frac{1}{\Gamma(j / 2)}\right)^{m} \operatorname{det} A,
$$

where for $N$ even

$$
A=\left[\alpha_{2 j-1,2 k}\right]_{j, k=1, \ldots, N / 2},
$$

while for $N$ odd

$$
A=\left[\left[\alpha_{2 j-1,2 k}\right]_{\substack{j=1, \ldots,(N+1) / 2 \\ k=1, \ldots,(N-1) / 2}}\left[\nu_{2 j-1}\right]_{j=1, \ldots,(N+1) / 2}\right] .
$$

Here the matrix elements are specified by

$$
\begin{aligned}
\alpha_{j, k} & =\int_{-\infty}^{\infty} d x \int_{-\infty}^{\infty} d y w_{m}(x) w_{m}(y) x^{j-1} y^{k-1} \operatorname{sgn}(y-x) \\
& =:\left\langle x^{j-1} y^{k-1} \operatorname{sgn}(y-x)\right\rangle_{x, y \in \mathrm{N}^{(m)}[0,1]}
\end{aligned}
$$

(recall we are using $\mathrm{N}^{(m)}[0,1]$ to denote the distribution of the product of $m$ standard Gaussian random variables) and

$$
\nu_{j}=\left\langle x^{j-1}\right\rangle_{x \in \mathrm{N}^{(m)}[0,1]} .
$$


Proof. According to the method of integration over alternate variables (see e.g. [24, Prop. 6.3.4]), for $N$ even

$$
\int_{L} w_{m}\left(\lambda_{1}\right) \cdots w_{m}\left(\lambda_{N}\right) \prod_{1 \leq j<k \leq N}\left(\lambda_{j}-\lambda_{k}\right)=\operatorname{Pf}\left[\alpha_{j, k}\right]_{j, k=1, \ldots, N} .
$$

But for $w_{m}(x)$ even, $\alpha_{2 j, 2 k}=\alpha_{2 j-1,2 k-1}=0$, showing that the entries of the Pfaffian vanish in a chequerboard pattern, allowing it to be written as the determinant (2.15). The case $N$ odd follows by appropriately modifying the method of integration over alternate variables [24, Exercises 6.3 q.1], and an analogous reduction of the resulting Pfaffian to a determinant of half the size.

Our next task is to evaluate the matrix elements (2.17) and (2.18).

Proposition 3. We have

$$
\alpha_{2 j-1,2 k}=\frac{1}{(2 \pi)^{m}} 2^{(j+k-1 / 2) m} G_{m+1, m+1}^{m+1, m}\left(1 \begin{array}{c}
5 / 2-j, \ldots, 5 / 2-j, 2 \\
1,1+k, \ldots, 1+k
\end{array}\right)
$$

and

$$
\nu_{2 j-1}=\left(\frac{1}{\sqrt{2 \pi}}\right)^{m}\left(\Gamma\left(j-\frac{1}{2}\right)\right)^{m}
$$

Thus, for $N$ even

$$
p_{N, N}^{P_{m}}=\left(\prod_{j=1}^{N} \frac{1}{\Gamma(j / 2)}\right)^{m} \operatorname{det}\left[G_{m+1, m+1}^{m+1, m}\left(1 \mid \begin{array}{c}
5 / 2-j, \ldots, 5 / 2-j, 2 \\
1,1+k, \ldots, 1+k
\end{array}\right)\right]_{j, k=1, \ldots, N / 2}
$$

while for $N$ odd

$$
\begin{aligned}
p_{N, N}^{P_{m}} & =\left(\prod_{j=1}^{N} \frac{1}{\Gamma(j / 2)}\right)^{m} \\
& \times \operatorname{det}\left[\left[G_{m+1, m+1}^{m+1, m}\left(1 \mid \begin{array}{c}
5 / 2-j, \ldots, 5 / 2-j, 2 \\
1,1+k, \ldots, 1+k
\end{array}\right)\right]_{\substack{j=1, \ldots,(N+1) / 2 \\
k=1, \ldots,(N+1) / 2}} \quad\left[\left(\Gamma\left(j-\frac{1}{2}\right)\right)^{m}\right]_{j=1, \ldots,(N+1) / 2}\right] .
\end{aligned}
$$

Proof. We first note that

$$
\alpha_{2 j-1,2 k}=2\left\langle x^{2 j-2} y^{2 k-1} \chi_{y>x}\right\rangle_{x, y \in \mathrm{N}^{(m)}[0,1]},
$$

where $\chi_{J}$ for $J$ true, $\chi_{J}=0$ otherwise. Recalling (2.12) and applying a simple change of variables shows

$$
\begin{aligned}
\alpha_{2 j-1,2 k}= & \frac{1}{(2 \pi)^{m}} 2^{(j+k-1 / 2) m} \\
& \times \int_{0}^{\infty} d x x^{j-3 / 2} G_{0, m}^{m, 0}\left(\left.x\right|_{0, \ldots, 0}\right) \int_{x}^{\infty} d y y^{k-1} G_{0, m}^{m, 0}\left(\left.y\right|_{0, \ldots, 0}\right) .
\end{aligned}
$$


Use of computer algebra gives

$$
\int_{x}^{\infty} d y y^{k-1} G_{0, m}^{m, 0}\left(\left.y\right|_{0, \ldots, 0}\right)=G_{1, m+1}^{m+1,0}\left(\left.x\right|_{0, k, \ldots, k}\right)
$$

and furthermore

$$
\begin{gathered}
\int_{0}^{\infty} d x x^{j-3 / 2} G_{0, m}^{m, 0}\left(\left.x\right|_{0, \ldots, 0}-G_{1, m+1}^{m+1,0}\left(\left.x\right|_{0, k, \ldots, k} \begin{array}{c}
1 \\
0, \ldots, 5 / 2-j, 2
\end{array}\right)\right. \\
=G_{m+1, m+1}^{m+1, m}\left(1 \mid \begin{array}{c}
5 / 2-j, \ldots, 5 \\
1,1+k, \ldots, 1+k
\end{array}\right)
\end{gathered}
$$

thus implying (2.19). The result (2.20) now follows by substituting (2.19) in (2.14) and straightforward simplification.

It is furthermore the case that

$$
\nu_{2 j-1}=\left(\frac{1}{\sqrt{2 \pi}} \int_{-\infty}^{\infty} x^{2 j-2} e^{-x^{2} / 2} d x\right)^{m},
$$

which implies (2.20). Substituting this and (2.20) in (2.14) in the case (2.16) and simplifying gives (2.22).

We remark that as well as occurring in the study of products of Gaussian random matrices, the Meijer- $G$ function also occurs in random matrix theory in the study of the value distribution of determinants [16, 21] and the study of the Cauchy two-matrix model [10, 11]. The limiting correlation kernels appearing in the latter works have been related to that for the ensemble of generalized Wishart matrices $P_{2}^{\dagger} P_{2}$ in [33].

\subsection{Evaluations}

Consider first the case $m=2$, and thus the product of two Gaussian matrices $X, Y$ say. Although we have no proof, high precision computer calculations indicate that the Meijer $G$-functions in (2.19) are all rational multiples of $\pi^{2}$, and furthermore the denominator of each is a power of 2 . For example, with $m=2, N=6$

$$
\left[G_{3,3}^{3,2}\left(1 \mid \begin{array}{c}
5 / 2-j, 5 / 2-j, 2 \\
1,1+k, 1+k
\end{array}\right)\right]_{j, k=1,2,3} \stackrel{?}{=} \pi^{2}\left[\begin{array}{ccc}
\frac{1}{2^{2}} & \frac{39}{2^{5}} & \frac{10335}{2^{13}} \\
\frac{3}{2^{5}} & \frac{435}{2^{10}} & \frac{72555}{2^{18}} \\
\frac{135}{2^{13}} & \frac{16695}{2^{18}} & \frac{15107715}{2^{25}}
\end{array}\right] .
$$

Assuming the validity of these forms, use of (2.21) and (2.22) then give the exact values

$$
\begin{aligned}
p_{2,2}^{X Y}=\frac{\pi}{2^{2}}, & p_{3,3}^{X Y} & =\frac{5 \pi}{2^{5}} \\
p_{4,4}^{X Y}=\frac{201 \pi^{2}}{2^{13}}, & p_{5,5}^{X Y} & =\frac{10013 \pi^{2}}{2^{20}} \\
p_{6,6}^{X Y}=\frac{64011585 \pi^{3}}{2^{36}}, & p_{7,7}^{X Y} & =\frac{31625532537 \pi^{3}}{2^{47}}
\end{aligned}
$$




\begin{tabular}{c||c}
$m$ & $p_{2,2}^{P_{m}}$ \\
\hline 2 & 0.7853981634 \\
3 & 0.8357987202 \\
4 & 0.8716118625 \\
5 & 0.8982590645 \\
6 & 0.9186258752 \\
7 & 0.9344692620 \\
8 & 0.9469484311 \\
9 & 0.9568694180 \\
10 & 0.9648135032
\end{tabular}

Table 1: First ten decimal places of the probability $p_{2,2}^{P_{m}}$ that the random matrix product $P_{m}=X_{m} X_{m-1} \cdots X_{1}$, with each $X_{i}$ a $2 \times 2$ standard Gaussian matrix, has all eigenvalues real.

The first of these has been derived in the recent work [34] (see also [40] and Section 2.4] below). Note that the case $m=2$ is special in that the corresponding weight function has the $K_{0}$ Bessel function form (2.13). We remark that the $K_{0}$ Bessel function also appears in other closed form evaluations in mathematical physics, in particular relating to the twodimensional Ising model [8, 7]. For an informative recent article relating to high precision computations and closed form evaluations we refer to [12].

Analysis of the corresponding numerical values, extended to $N=25$ and formed into the ratio

$$
\frac{p_{2 j-1,2 j-1}^{X Y} p_{2 j+1,2 j+1}^{X Y}}{\left(p_{2 j, 2 j}^{X Y}\right)^{2}}
$$

indicates that for large $j$ this has the limit value $\pi / 4$ and that for large $N$

$$
p_{N, N}^{X Y} \sim(\pi / 4)^{N^{2} / 2}
$$

This is a faster decay rate than seen in (1.3) for $p_{N, N}^{Y^{-1} X}$ (compare bases $(\pi / 4)^{1 / 2} \approx 0.886$ and $\left.(e / 4)^{1 / 4} \approx 0.907\right)$. In the next subsection an analytic derivation of (2.26) will be given, as will the leading large $N$ form of $p_{N, N}^{P_{m}}$ for general $m$.

We now turn our attention to the case $N=2$. We read off from (2.21) that

$$
p_{2,2}^{P_{m}}=\frac{1}{\pi^{m / 2}} G_{m+1, m+1}^{m+1, m}\left(1 \mid \begin{array}{c}
3 / 2, \ldots, 3 / 2,2 \\
1,2, \ldots, 2
\end{array}\right)
$$

In Table 1 we list the corresponding numerical values up to $m=10$. High precision computation was used, but no evidence of special arithmetic structures was found for $m>2$. Analysis of the ratio $\left(1-p_{2,2}^{P_{m+1}}\right) /\left(1-p_{2,2}^{P_{m}}\right)$ for successive $m$ up to 16 gave values $\approx 0.82$ but slowly increasing in the third decimal, so evidence for an exponential approach to unity was inconclusive. 


\subsection{Leading large $N$ form of $p_{N, N}^{P_{m}}$}

The known analytic result (1.3) for $m=1$ and the numerical conjecture (2.26) for $m=2$ both exhibit a Gaussian decay in $N$ for $p_{N, N}^{P_{m}}$, but with different bases $b_{m}, b_{1}<b_{2}$. It is possible to establish a Gaussian decay for each $m$, and furthermore to determine $b_{m}$.

To begin, we know from [24, eq. (4.186)] that

$$
\log \prod_{j=1}^{N} \Gamma(j / 2) \sim \frac{N^{2}}{4} \log \frac{N}{2}-\frac{3}{8} N^{2}+O(N \log N) .
$$

Substituting this in (2.8) and changing variables $\lambda_{l} \mapsto\left(c_{m / 2} N\right)^{m / 2} \lambda_{l}$ shows

$$
\begin{aligned}
\log p_{N, N}^{P_{m}} & \sim \frac{3 m N^{2}}{8}+\frac{N^{2} m}{4} \log 2 c_{m / 2} \\
& +\log \int_{L} \prod_{l=1}^{N} w_{m}\left(\left(c_{m / 2} N\right)^{m / 2} \lambda_{l}\right) \prod_{1 \leq j<k \leq N}\left(\lambda_{j}-\lambda_{k}\right) d \lambda_{1} \cdots d \lambda_{N}+O(N \log N) .
\end{aligned}
$$

Furthermore, since $N$ is large, we can use knowledge of the large argument form of the Meijer $G$-function in (2.12) as given in e.g. [6, pg. 12] to write

$$
w_{m}\left(N^{m / 2} \lambda\right)=e^{-m c_{m / 2} N|\lambda|^{2 / m} / 2+O(\log N)},
$$

allowing us to replace the logarithm of the integral in the final line of (2.28) by

$$
I_{m, N}:=\log \int_{L} \prod_{l=1}^{N} e^{-m c_{m / 2} N\left|\lambda_{l}\right|^{2 / m} / 2} \prod_{1 \leq j<k \leq N}\left(\lambda_{j}-\lambda_{k}\right) d \lambda_{1} \cdots d \lambda_{N} .
$$

It is known rigorously (see e.g. [36, eq. (11.1.22)]) that

$$
\log I_{m, N} \sim N^{2} \mathcal{E}
$$

where with $\rho(x)$ denoting the scaled density of the Coulomb gas model implied by (2.29), supported on the single interval $(-a, a)$,

$$
\mathcal{E}=-\int_{-a}^{a} V(x) \rho(x) d x+\frac{1}{2} \int_{-a}^{a} d x_{1} \rho\left(x_{1}\right) \int_{-a}^{a} d x_{2} \rho\left(x_{2}\right) \log \left|x_{1}-x_{2}\right|
$$

with $V(x)=m c_{m / 2}|x|^{2 / m} / 2$. Moreover $\rho(x)$ is such that (2.31) is minimised, giving rise to the terminology 'the equilibrium problem' with $\rho(x) d x$ the equilibrium measure, while the one-body Boltzmann factor $e^{-V(x)}$ with $V$ proportional to $|x|^{\alpha}$ is referred to as the Freud weight. 
Proposition 4. Choose $c_{m / 2}$ in (2.29) so that $\rho(x)$ is supported on $(-1,1)$. Then we have

$$
\mathcal{E}=-\frac{1}{2} \log 2-\frac{3 m}{8}
$$

Proof. We know from [38] that choosing

$$
\frac{m c_{m / 2}}{2}=\frac{\Gamma(1 / m) \Gamma(1 / 2)}{2 \Gamma(1 / m+1 / 2)}
$$

implies that $\rho(x)$ is supported on $(-1,1)$, that it has the explicit value

$$
\rho(x)=\frac{1}{m \pi} \int_{|x|}^{1} \frac{u^{1 / m-1}}{\sqrt{u^{2}-t^{2}}} d u,
$$

and furthermore

$$
\int_{-a}^{a} d x \rho(x) \log |x-y|=\frac{m c_{m / 2}}{2}|y|^{2 / m}-\log 2-\frac{m}{2}
$$

It follows from (2.35) substituted in (2.31) that

$$
\begin{aligned}
\mathcal{E} & =-\frac{1}{2}\left(\log 2+\frac{m}{2}\right)+\frac{m c_{m / 2}}{4} \int_{-1}^{1} \rho(x)|x|^{1 / m} d x \\
& =-\frac{1}{2}\left(\log 2+\frac{3 m}{4}\right)
\end{aligned}
$$

where the second line follows upon use of the explicit form of $\rho(x)$ (2.34), (2.33), and the Euler beta integral.

Substituting (2.32) in (2.30), substituting the result of this in the second line of (2.28) and making use of (2.33) in the first shows that

$$
\log p_{N, N}^{P_{m}} \sim N^{2}\left(-\frac{1}{2} \log 2+\frac{m}{4} \log \left(\frac{\Gamma(1 / m+1) \Gamma(1 / 2)}{\Gamma(1 / m+1 / 2)}\right)\right)
$$

or equivalently

$$
p_{N, N}^{P_{m}} \underset{N \rightarrow \infty}{\sim} b_{m}^{N^{2}}, \quad b_{m}=\frac{1}{\sqrt{2}}\left(\frac{\Gamma(1 / m+1) \Gamma(1 / 2)}{\Gamma(1 / m+1 / 2)}\right)^{m / 4} .
$$

Substituting $m=1$ we reclaim the leading large $N$ form implied by (1.3),$p_{N, N}^{P_{1}} \sim 2^{-N^{2} / 4}$, while setting $m=2$ we obtain the conjectured form (2.26). We can check from (2.37) that $b_{m}$ in (2.38) is an increasing function of $m$ which tends to unity as $m \rightarrow \infty$. This latter feature is consistent with all eigenvalues being real in this limit, a topic we now turn to from a different perspective in the case $N=2$, before returning to (2.21) and (2.22) to give a demonstration for general $N$. 


\subsection{Alternative expression for $p_{2,2}^{P_{m}}$}

As indicated, we conclude by deriving an alternative expression to (2.27) for $p_{2,2}^{P_{m}}$, which allows us to both read off the exact value of $p_{2,2}^{P_{2}}$, and to give some insight into the phenomenon $p_{2,2}^{P_{m}} \rightarrow 1$ as $m \rightarrow \infty$ observed through simulation in [34], and in our list of exact decimal values in Table 1. We then make use of (2.21) and (2.22) to show that $p_{N, N}^{P_{m}} \rightarrow 1$ as $m \rightarrow \infty$ for general $N \geq 2$.

Proposition 5. With the notation $\mathrm{N}^{(m)}[0,1]$ for the distribution of $m$ standard Gaussian random variables as used above we have

$$
p_{2,2}^{P_{m}}=\frac{1}{2}\left(\sqrt{\frac{\pi}{2}}\right)^{m-1}\left\langle\sqrt{x^{2}+y^{2}}\right\rangle_{x, y \in \mathrm{N}^{(m-1)}[0,1]} .
$$

Proof. We seek a formula for $\alpha_{1,2}$ as defined by (2.17) different to that in (2.19). Now

$$
\alpha_{1,2}=\left\langle(y-x) \chi_{y>x}\right\rangle_{x, y \in \mathrm{N}^{(m)}[0,1]}=\int_{0}^{\infty} d s s \int_{-\infty}^{\infty} d x w_{m}(x) w_{m}(x+s) .
$$

According to the definition (2.7), upon carrying out the integration over $x_{m}$,

$$
w_{m}(x)=\left(\frac{1}{\sqrt{2 \pi}}\right)^{m} \int_{-\infty}^{\infty} d x_{1} \cdots \int_{-\infty}^{\infty} d x_{m-1} \frac{1}{\left|X^{(m-1)}\right|} e^{-\sum_{j=1}^{m-1} x_{j}^{2} / 2} e^{-x^{2} /\left(2\left(X^{(m-1)}\right)^{2}\right)},
$$

where $X^{(m-1)}:=\prod_{l=1}^{m-1} x_{l}$. Hence

$$
\begin{aligned}
& \int_{-\infty}^{\infty} d x w_{m}(x) w_{m}(x+s) \\
& =\frac{1}{\sqrt{2 \pi}}\left\langle e^{-s^{2} /\left(2\left(\left(X^{(m-1)}\right)^{2}+\left(Y^{(m-1)}\right)^{2}\right)\right)} \sqrt{\left.\left.\left(X^{(m-1)}\right)^{2}+\left(Y^{(m-1)}\right)^{2}\right)\right)}\right\rangle_{x_{l}, y_{l} \in \mathrm{N}[0,1](l=1, \ldots, m-1)} .
\end{aligned}
$$

Substituting in (2.40) allows the integration over $s$ to be carried out, showing that

$$
\alpha_{1,2}=\frac{1}{\sqrt{2 \pi}}\left\langle\sqrt{\left.\left.\left(X^{(m-1)}\right)^{2}+\left(Y^{(m-1)}\right)^{2}\right)\right)}\right\rangle_{x_{l}, y_{l} \in \mathrm{N}[0,1](l=1, \ldots, m-1)} .
$$

Substituting this in (2.14) with $m=2$ and recalling the definition of $\mathrm{N}^{(m-1)}[0,1]$ gives (2.39).

According to (2.39)

$$
\begin{aligned}
& p_{2,2}^{P_{1}}=\frac{1}{\sqrt{2}} \\
& p_{2,2}^{P_{2}}=\frac{1}{2} \sqrt{\frac{\pi}{2}}\left\langle\sqrt{x^{2}+y^{2}}\right\rangle_{x, y \in \mathrm{N}[0,1]}=\frac{\pi}{4},
\end{aligned}
$$


where the second equality follows upon using polar coordinates. The result (2.41) is the special case $N=2$ of Edelman's result (1.3), while (2.42) is the first of the results in (2.25), which as already remarked has been proved recently in [34] using different integration methods.

Using (2.39) we can get some insight into the $m \rightarrow \infty$ behaviour. Thus one has that

$$
\left(\sqrt{\frac{\pi}{2}}\right)^{m-1}\langle|x|\rangle_{x, y \in \mathrm{N}^{(m-1)}[0,1]}=1, \quad\left(\sqrt{\frac{\pi}{2}}\right)^{m-1}\left\langle x^{2}\right\rangle_{x, y \in \mathrm{N}^{(m-1)}[0,1]}=\left(\sqrt{\frac{\pi}{2}}\right)^{m-1}
$$

telling us that the variance of the random variable $\prod_{p=1}^{m-1}\left|x_{l}\right|$ for $x_{l} \in \mathrm{N}[0,1]$ is exponentially larger than the mean. Thus, as in vividly demonstrated by Monte Carlo simulation, to leading order the random variables $x$ and $y$ in (2.39) are independent (typically simulated values of $|x|$ and $|y|$ are close to zero, with occasional large values which contribute most to the final average occurring independently). This shows that for large $m$

$$
p_{2,2}^{P_{m}} \rightarrow \frac{1}{2}\left(\left(\sqrt{\frac{\pi}{2}}\right)^{m-1}\langle|x|\rangle_{x \in \mathrm{N}^{(m-1)}[0,1]}+\left(\sqrt{\frac{\pi}{2}}\right)^{m-1}\langle|y|\rangle_{y \in \mathrm{N}^{(m-1)}[0,1]}\right)=1,
$$

in agreement with the result of Table 1 and the simulations of [34].

In fact the formulas (2.21) and (2.22) can be used to show that more generally, for any $N \geq 2, p_{N, N}^{P_{m}} \rightarrow 1$ as $m \rightarrow \infty$, in agreement with the extended simulations of [34].

Proposition 6. We have

$$
\lim _{m \rightarrow \infty}\left(\frac{1}{\Gamma(j-1 / 2) \Gamma(k)}\right)^{m} G_{m+1, m+1}^{m+1, m}\left(1 \mid \begin{array}{c}
3 / 2-j, \ldots, 3 / 2-j, 1 \\
0, k, \ldots, k
\end{array}\right)= \begin{cases}1, & j \leq k \\
0, & j>k\end{cases}
$$

and thus for $N \geq 2$

$$
p_{N, N}^{P_{m}} \rightarrow 1 \quad \text { as } \quad m \rightarrow \infty .
$$

Proof. $\quad$ Since from the definition (2.11)

$$
G_{m+1, m+1}^{m+1, m}\left(1 \mid \begin{array}{c}
3 / 2-j, \ldots, 3 / 2-j, 1 \\
0, k, \ldots, k
\end{array}\right)=G_{m+1, m+1}^{m+1, m}\left(\begin{array}{c}
1 \\
5 / 2-j, \ldots, 5 / 2-j, 2 \\
0, k, \ldots, k
\end{array}\right)
$$

we see from (2.21) and (2.22) that (2.44) follows from (2.43), so it remains to establish the latter.

Now (2.11) gives

$$
G_{m+1, m+1}^{m+1, m}\left(1 \mid \begin{array}{c}
3 / 2-j, \ldots, 3 / 2-j, 1 \\
0, k, \ldots, k
\end{array}\right)=-\frac{1}{2 \pi i} \int_{C} \frac{\left(\Gamma(k-s) \Gamma\left(j-\frac{1}{2}+s\right)\right)^{m}}{s} d s
$$

where $C$ can be taken to be a contour starting at $-i \infty$, passing through the real axis within the interval $\left(\frac{1}{2}-j, 0\right)$ and finishing at $i \infty$. Changing variables $s \rightarrow s / m$ we see that for large $m$

$$
\left(\Gamma\left(k-\frac{s}{m}\right) \Gamma\left(j-\frac{1}{2}+\frac{s}{m}\right)\right)^{m} \rightarrow\left(\Gamma(k) \Gamma\left(j-\frac{1}{2}\right)\right)^{m} e^{-s(\Psi(k)-\Psi(j-1 / 2))},
$$


where $\Psi(z)$ denotes the digamma function. But for the contour $C$ running from $-i \infty$ to $i \infty$ and passing to the left of the origin, and with $r$ real

$$
-\frac{1}{2 \pi i} \int_{C} \frac{e^{-s r}}{s} d s=\left\{\begin{array}{ll}
1, & r>0 \\
0, & r<0
\end{array},\right.
$$

as is seen by closing the contours to the right $(r>0)$, left $(r<0)$. The result now follows since $\Psi(k)-\Psi(j-1 / 2))>0$ for $k \geq j$ while this quantity is less than zero for $k<j$.

\section{Acknowledgements}

This work was supported by the Australian Research Council. I thank Gernot Akemann for comments on the first draft, and Mario Kieburg for sending me a copy of [29].

\section{References}

[1] K. Adhikari, N.K. Reddy, T.R. Reddy, and K. Saha, Determinantal point processes in the plane from products of random matrices, arXiv:1308.6817.

[2] G. Akemann and Z. Burda, Universal microscopic correlations for products of independent Ginibre matrices, J. Phys. A 45 (2012), 465210 (18pp).

[3] G. Akemann, J. Ipsen, and M. Kieburg, Products of rectangular random matrices: singular values and progressive scattering, arXiv:1307.7560, 2013.

[4] G. Akemann, M. Kieburg, and L. Wei, Singular value correlation functions for products of Wishart random matrices, J. Phys. A 46 (2013), 275205 (22pp).

[5] G. Akemann, M.J. Phillips, and H.-J. Sommers, The chiral Gaussian two-matrix ensemble of real asymmetric matrices, J. Phys. A 43 (2009), 085211 (29pp).

[6] G. Akemann and E Strahov, Hole probabilities and overcrowding estimates for products of complex Gaussian matrices, J. Stat. Phys. 151 (2013), 987-1003.

[7] D.H. Bailey, J.M. Borwein, D. Broadhurst, and M.L. Glasser, Elliptic integral evaluations of Bessel moments and applications, J.Phys.A 41 (2008), 205203 (46pp).

[8] D.H. Bailey, J.M. Borwein, and R.E. Crandall, Integrals of the Ising class, J.Phys.A 39 (2006), 12271-12302.

[9] G. Bergqvist and P.J. Forrester, Rank probabilities for real random $n \times n \times 2$ tensors, Electronic Comm. Probab. 16 (2011), 1655.

[10] M. Bertola, M. Gekhtman, and J. Szmigielski, The Cauchy two-matrix model, Commun. Math. Phys. 287 (2009), 983-1014. 
[11] _ Cauchy-Laguerre two-matrix model and the Meijer-G random point field, arXiv:1211.5369, 2012.

[12] J.M. Borwein and R.E. Crandall, Closed forms: What they are and why we care, Notices Amer. Math. Soc. 60 (2013), 50-65.

[13] P. Bougerol and J. Lacroix, Products of random matrices with applications to Schrödinger operators (P. Huber and M. Rosenblatt, eds.), Progress in probability and statistics, vol. 8, Birkjäuser, Boston, 1985.

[14] Z. Burda, R.A. Janik, and B. Waclaw, Spectrum of the product of independent random Gaussian matrices, Phys. Rev. E 81 (2010), 041132.

[15] Z. Burda, A. Jarosz, G. Livan, M.A. Nowak, and A. Swiech, Eigenvalues and singular values of products of rectangular Gaussian random matrices, Acta Phys. Polon. B42 (2011), 939-985.

[16] G.M. Cicuta and M.L. Mehta, Probability density of determinants of random matrices, J.Phys.A 33 (2000), 8029-8035.

[17] J.E. Cohen, H. Kesten, and C.M. Newman (eds.), Random matrices and their applications, Contemporary Mathematics, vol. 50, American Mathematical Society, Providence, RI, 1986.

[18] A. Comtet, J.M. Luck, C. Texier, and Y. Tourigny, The Lyapunov exponent of products of random $2 \times 2$ matrices close to the identity, J. Stat. Phys. 150 (2013), 13-65.

[19] A. Comtet, C. Texier, and Y. Tourigny, Products of random matrices and generalized quantum point scatters, J. Stat. Phys. 140 (2010), 427-466.

[20] A. Crisanti, G. Paladin, and A. Vulpiani, Products of random matrices, Random matrices and their applications, Springer series in solid-state sciences, vol. 104, SpringerVerlag, Berlin Heidelberg, 1993.

[21] R. Delannay and G. Le Caer, Distribution of the determinant of a random realsymmetrix matrix from the gaussian orthogonal ensemble, Phys. Rev. E 62 (2000), $1526-1536$.

[22] A. Edelman, The probability that a random real Gaussian matrix has $k$ real eigenvalues, related distributions, and the circular law, J. Multivariate. Anal. 60 (1997), 203-232.

[23] A. Edelman, E. Kostlan, and M. Shub, How many eigenvalues of random matrix are real?, J. Amer. Math. Soc. 7 (1994), 247-267.

[24] P.J. Forrester, Log-gases and random matrices, Princeton University Press, Princeton, NJ, 2010. 
[25] L Lyapunov exponents for products of complex Gaussian random matrices, J. Stat. Phys. 151 (2012), 796-808.

[26] P.J. Forrester and A. Mays, Pfaffian point process for the Gaussian real generalised eigenvalue problem, Prob. Th. Related Fields 154 (2012), 1-47.

[27] P.J. Forrester and T. Nagao, Eigenvalue statistics of the real Ginibre ensemble, Phys. Rev. Lett. 99 (2007), 050603.

[28] J.R. Ipsen, Products of independent quaternion Ginibre matrices and their correlation functions, J. Phys. A 46 (2013), 265201 (16pp).

[29] J.R. Ipsen and M. Kieburg, Weak commutation relations and eigenvalue statistics of products of rectangular random matrices, arXiv:1310.4154.

[30] E. Kanzieper and G. Akemann, Statistics of real eigenvalues in Ginibre's ensemble of random real matrices, Phys. Rev. Lett. 95 (2005), 230201.

[31] E. Kanzieper and N. Singh, Non-Hermitean Wishart random matrices, J. Math. Phys. 51 (2010), 103510 (28pp).

[32] V. Kargin, On the largest Lyapunov exponent for products of Gaussian random matrices, arXiv:1302.2153, 2013.

[33] A.B.J. Kujlaars and L. Zhang, Singular values of products of Gaussian random matrices, multiple orthogonal polynomials and hard edge scaling limits, arXiv:1308.1003, 2013.

[34] A. Lakshminarayan, On the number of real eigenvalues of products of random matrices and an application to quantum entanglement, J. Phys. A 46 (2013), 152003.

[35] J.C. Osborn, Universal results from an alternative random matrix model for QCD with a baryon chemical potential, Phys. Rev. Lett. 93 (2004), 222001.

[36] L. Pastur and M. Shcherbina, Eigenvalue distribution of large random matrices, American Mathematical Society, Providence, RI, 2011.

[37] K.A. Penson and K. Zyczkowski, Product of Ginibre matrices: Fuss-Catalan and Raney distributions, Phys. Rev. E 83 (2011), 061118.

[38] E.B. Saff and V. Totik, Logarithmic potentials with external fields, Springer, Berlin, 1997.

[39] H. Schmidt, Disordered one-dimensional crystals, Phys. Rev. 105 (1957), 425-441.

[40] K.V. Shuddhodan, M.S. Ramkarthik, and A. Lakshminarayan, Entanglement optimizing mixtures of two-qubit states, J.Phys.A 44 (2011), 345301 (12pp). 